hep-th/9904142

PUPT-1859

IAS-SNS-HEP-99-37

\title{
Comments on the IIA NS5-brane
}

\author{
Shiraz Minwalla ${ }^{1}$ \\ Department of Physics, Princeton University \\ Princeton, NJ 08544, USA \\ and \\ Nathan Seiberg ${ }^{2}$ \\ School of Natural Sciences, Institute for Advanced Study \\ Olden Lane, Princeton, NJ 08540, USA
}

\begin{abstract}
We study $N$ coincident IIA NS5-branes at large $N$ using supergravity. We show that the absorption cross section for gravitons in this background does not vanish at zero string coupling for energies larger than $\frac{m_{s}}{\sqrt{N}}\left(m_{s}\right.$ is the string scale). Using a holographic description of the intrinsic theory of the IIA NS5-branes, we find an expression for the two point function of the stress energy tensor, and comment on its structure.
\end{abstract}

April 1999

\footnotetext{
1 minwalla@princeton.edu

2 seiberg@sns.ias.edu
} 


\section{Introduction}

In this note we study $N$ coincident NS5-branes [1] in type II theory. In the limit $g_{0} \rightarrow 0, m_{s}$ fixed (where $g_{0}$ is the asymptotic value of the string coupling and $m_{s}$ is the string mass) the theory is free in the bulk. However, modes living on the 5-brane continue to interact amongst themselves, while decoupling from the bulk, defining a mysterious nongravitational six-dimensional theory [2] (this construction was motivated by [3]), sometimes referred to as the little string theory. Upon compactification, this theory inherits the $T$ duality of type II string theory and is therefore nonlocal at the scale $m_{s}$.

Maldacena and Strominger [4] studied the system of $N$ non-extremal coincident NS5branes with excitation energy density $\mu m_{s}^{6}$. The classical solution for this configuration possesses an asymptotically flat region, which turns into a tube on approaching the 5brane. On descending down the tube one encounters the horizon of the black branes. The local string coupling outside the horizon is everywhere less than $\sqrt{\frac{N}{\mu}}$, and all curvatures in this region are less than $\frac{m_{s}}{\sqrt{N}}$. Therefore, when $\mu \gg N \gg 1$ semi-classical gravity is accurate outside the horizon. In particular, the brane Hawking radiates at temperature $\frac{1}{2 \pi} \frac{m_{s}}{\sqrt{N}}$, and modes behind the horizon continue to couple to modes in the tube even for $g_{0}=0$.

This fact was interpreted in [5] as a manifestation of holography in the underlying string theory. In particular, the decoupled theory of the NS5-branes is the holographic projection, along the lines of [6], of the near horizon geometry ( $g_{0} \rightarrow 0$ limit) including the long tube日. It was further proposed in [5] that some of the observables of the NS5-brane theory are the on-shell particles (vertex operators in the string theory limit) in the bulk. Unlike the situation in $A d S$, our geometry admits the definition of an $S$ matrix. Hence, off-shell six-dimensional "Green's functions" of these observables are identified as the $S$ matrix elements of the corresponding higher dimensional bulk particles.

It should be emphasized that the boundary of the near horizon geometry of the Euclidean NS5-brane is $R^{6} \times S^{3}$, and reduces to $R^{6}$ only after a Kaluza Klein reduction on the $S^{3}$. This is in contrast with the near horizon geometry of, say, the Euclidean M5-brane, whose boundary is $S^{6}$ rather than $S^{6} \times S^{4}$ (because the ratio of the size of the $S^{4}$ to that of the $S^{6}$ goes to zero on approaching the boundary).

1 Similar ideas have been suggested by various people, including C.V. Johnson, J. Maldacena and A. Strominger. 
The Euclidean near horizon geometry of the NS5-brane consists of a semi-infinite tube, capped at the bottom by (Euclidean) $A d S_{7} \times S^{4}$. Let the analytic continuation of this space to Lorentzian signature be denoted by $E$. $E$ possesses a future horizon $H^{+}$and a past horizon $H^{-}$each at finite affine distance, and so is geodesically incomplete. Its metric may be completed (see Appendix D) by gluing together a new copy of $E$ at each of the two original horizons, and then continuing this procedure indefinitely (just as the AdS cylinder may be constructed by gluing together copies of Poincare patches at their horizons). The resulting Penrose diagram is shown in fig. 1, and contains an infinite number of pairs of $\mathcal{I}_{i}^{ \pm} \mathrm{s}$, differentiated in our notation by the subscript $i . \mathcal{I}_{i}^{ \pm}$, together with the horizons $H_{i}^{ \pm}$ constitute the boundary of the $i^{\text {th }}$ wedge $E_{i}$. It is not clear to us whether this analytic continuation is physically relevante.

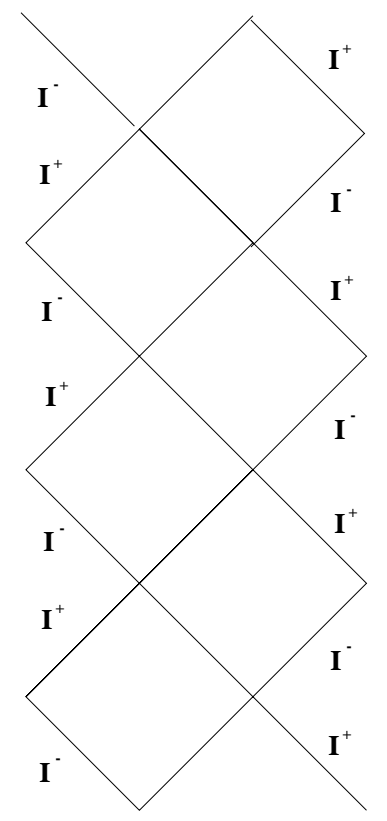

Fig. 1: The Penrose diagram of the geodesic completion of the metric of the NS5-brane.

The correlation functions defined in [5] in terms of $S$ matrix elements are naturally found in momentum space. However, it was pointed out by Peet and Polchinski [7] that these answers cannot be Fourier transformed to coordinate space, and therefore the little string theory is nonlocal. The reason for that is that the momentum space answers are obtained after a momentum dependent multiplicative renormalization with the characteristic scale $\frac{m_{s}}{\sqrt{N}}$. We will return to this below.

2 We thank O. Aharony and T. Banks for a useful discussion on this point. 
Aharony and Banks [8] computed the entropy of the little string theory as a function of energy $\omega$ using its DLCQ definition [9,10] and found $\frac{6 \sqrt{N} \omega}{m_{s}}$, in agreement with the Bekenstein-Hawking entropy computed in [四. This entropy formula suggests that the number of states in the system grows rapidly with energy, thus explaining why the typical momentum space correlation function cannot be Fourier transformed [7]. It also reproduces the scale of nonlocality as $\frac{m_{s}}{\sqrt{N}}$.

In this paper we study the propagation of a mode of the graviton, which propagates like a minimal scalar in the supergravity background of $N$ coincident IIA NS5-branes. Its $S$ matrix elements are interpreted as the correlation functions of the energy momentum tensor of the little string theory. In section 2 we discuss the validity of the supergravity approximation, define variables, and set up our equations. In section 3 we compute the absorption cross section of a graviton incident onto the 5-branes from the tube, and note that it is nonzero at $g_{0}=0$ for energies larger than $\frac{m_{s}}{\sqrt{N}}$. This result strengthens that of [4]. In section 4 we follow [5] to derive an expression for the two point function of the energy momentum tensor of the little string theory, and comment on its structure. In section 5 we explain the relation between our results and previous calculations. In Appendix A we estimate the high energy behavior of the two point function of section 4 using the WKB approximation. In Appendix B we develop a low energy expansion for the two point function. In Appendix $\mathrm{C}$ we study a simple toy model, similar in some ways to the NS5-brane. In Appendix D we discuss the causal structure of the geodesic completion of the near horizon geometry of the NS5-brane.

\section{The Setup}

\subsection{The Background Metric}

The classical string frame background corresponding to $N$ coincident extremal NS5branes is

$$
\begin{aligned}
& d s^{2}=d x_{6}^{2}+\left(1+\frac{N}{m_{s}^{2} r^{2}}\right)\left(d r^{2}+r^{2} d \Omega_{3}^{2}\right) \\
& e^{2 \Phi}=g_{0}^{2}\left(1+\frac{N}{m_{s}^{2} r^{2}}\right) .
\end{aligned}
$$

This background possesses an asymptotically flat region $r \gg \frac{\sqrt{N}}{m_{s}}$ connected to a semiinfinite flat tube $r \ll \frac{\sqrt{N}}{m_{s}}$, with the topology of $R^{+} \times S^{3} \times R^{6}$ (these factors are parametrized by $r, \Omega$ and $x_{6}$ respectively). 
In this paper we will restrict our attention to the IIA version of the little string theory. Its moduli space of vacua is $\frac{\left(R^{4} \times S^{1}\right)^{N}}{S_{N}}$ corresponding in $\mathrm{M}$ theory to the positions of the $N$ M5-branes in the transverse space and on the $11^{\text {th }}$ circle. (2.1) is the solution that corresponds to stacking all branes at the origin in the $R^{4}$, but smearing them evenly over the $S^{1}$, and therefore does not correspond to any true vacuum (single point in moduli space) of the theory.

We wish to study the theory at the most singular point in its moduli space, where all the branes are on top of each other both in $R^{4}$ and $S^{1}$. The corresponding classical background is most conveniently written in eleven dimensions [11]

$$
\begin{gathered}
d s^{2}=A^{-\frac{1}{3}} d x_{6}^{2}+A^{\frac{2}{3}}\left(d x_{11}^{2}+d r^{2}+r^{2} d \Omega_{3}^{2}\right) \\
A=1+\sum_{n=-\infty}^{\infty} \frac{N \pi l_{p}^{3}}{\left[r^{2}+\left(x_{11}-2 \pi n R_{11}\right)^{2}\right]^{\frac{3}{2}}} .
\end{gathered}
$$

The $11^{\text {th }}$ dimension is compact: $x_{11}=x_{11}+2 \pi R_{11}$ and $l_{p}$ is the eleven-dimensional Planck length. For $r \gg R_{11}$ the summation above may be replaced by an integral and we recover (2.1), on dimensional reduction and transforming to the string frame. For $r \ll R_{11}$ a single term in the summation in (2.3) dominates, and (2.2) reduces to the near horizon of $N$ stacked M5-branes. Therefore (2.2) represents asymptotic flat space connected to $A d S_{7} \times S^{4}$ by a long tube.

\subsection{Validity of Supergravity}

We start by considering the general case of $\mathrm{M}$ theory compactified on a circle, whose physical radius varies in an arbitrary spatially dependent fashion in the ten non-compact directions. We then specialize to M theory on (2.2).

When the physical length $r_{11}$ of the M theory circle is much larger than $l_{p}$ (the elevendimensional region) eleven-dimensional supergravity is valid for energies and curvatures much smaller than $\frac{1}{l_{p}}$. When $r_{11} \ll l_{p}$ (the ten-dimensional region) the local IIA string coupling $g=\left(\frac{r_{11}}{l_{p}}\right)^{\frac{3}{2}}$ is small, and string perturbation theory is valid. It reduces to IIA supergravity for energies and curvatures much smaller than the string scale. Under these conditions proper energies are smaller than $m_{e f f}=\frac{g}{r_{11}} \ll \frac{1}{r_{11}}$. Therefore, no Kaluza Klein modes on the $11^{\text {th }}$ circle are excited, and IIA supergravity is identical to eleven-dimensional supergravity. 
In summary, eleven-dimensional supergravity may be used over all space for energies $\omega \ll \frac{1}{l_{p}}, m_{s}$ provided curvatures are small in string units in the ten-dimensional region, and in Planck units in the eleven-dimensional region.

Throughout this paper $g_{0}$ is taken to be very small, so that $R_{11} \ll l_{p} \ll \frac{1}{m_{s}}$ and the energy condition above is satisfied if $\omega \ll m_{s}$. In the ten-dimensional region in (2.2), $r \gg R_{11} \sqrt{N}$, the curvatures in string units are always less than $\frac{1}{\sqrt{N}}$. Curvatures in the eleven-dimensional region $r \ll R_{11} \sqrt{N}$ are always less than $N^{-\frac{1}{3}}$ in Planck units.

Hence $11 \mathrm{~d}$ supergravity is valid in $(2.2)$ for $\omega \ll m_{s}$ and $N \gg 1$. Therefore, we study the theory in the 't Hooft limit $m_{s} \rightarrow \infty, N \rightarrow \infty$ with $\frac{m_{s}}{\sqrt{N}}$ fixed 3 . In this limit classical supergravity is valid at all energies.

\subsection{Qualitative motion}

We focus initially on the tube region, $R_{11} \ll r \ll \frac{\sqrt{N}}{m_{s}}$, where the expression for $A$ (2.3) simplifies to $A \approx \frac{N}{m_{s}^{2} r^{2}}$. A mode of energy $\omega$ at asymptotic infinity has proper energy $\omega A^{\frac{1}{6}}$. The local proper radius of the $11^{t h}$ circle is $r_{11}=R_{11} A^{\frac{1}{3}}$. The local value of the string coupling $g$ is $g_{0} A^{\frac{1}{2}}$. The local effective string tension (proper energy per unit proper length) is given by $m_{\text {eff }}^{2}=m_{s}^{2} A^{\frac{1}{3}}$.

The proper energy of a Kaluza Klein excitation in the $11^{\text {th }}$ direction is $\frac{1}{r_{11}}=\frac{1}{R_{11} A^{\frac{1}{3}}}$. This corresponds to energy at infinity $\omega=\frac{1}{R_{11} A^{\frac{1}{2}}}=\frac{r}{R_{11}} \frac{m_{s}}{\sqrt{N}}$. Therefore, Kaluza Klein modes are excited at the bottom of the tube for energies larger than $\frac{m_{s}}{\sqrt{N}}$.

A graviton with polarization parallel to the brane propagating in (2.2) with no momentum along the $S^{3}$ or the brane directions obeys at the quadratic level the equation of motion of a minimally coupled scalar [12]. Such a graviton couples to a particular polarization of the stress energy tensor on the brane world volumel 1 . We denote it by $\phi$ and study its propagation in (2.2). In the tube $\left(\frac{\sqrt{N}}{m_{s}} \gg r \gg R_{11}\right)$ we change coordinates to $z=\frac{\sqrt{N}}{m_{s}} \ln \left(\frac{r m_{s}}{\sqrt{N}}\right)$ and ignore variations in the $x_{11}$ direction to find the string frame metric and coupling constant

$$
\begin{gathered}
d s^{2}=d x_{6}^{2}+d z^{2}+\frac{N}{m_{s}^{2}} d \Omega_{3}^{2} \\
g=g_{0} e^{-\frac{z m_{s}}{\sqrt{N}}} .
\end{gathered}
$$

3 This is the usual 't Hooft limit for the low energy gauge theory of the IIB little string theory.

4 Upon compactification the theory on the brane has more than one "stress tensor" [2,5] and this is one of them. 
The Einstein frame metric is

$$
d s^{2}=e^{\frac{z m_{s}}{2 \sqrt{N}}}\left(d x_{6}^{2}+d z^{2}+\frac{N}{m_{s}^{2}} d \Omega_{3}^{2}\right)
$$

and the quadratic action for $\phi$ is

$$
S=\frac{(2 \pi)^{5}}{\kappa_{10}^{2}} \int d^{10} x e^{\frac{2 z m_{s}}{\sqrt{N}}}\left[\left(\partial_{0} \phi\right)^{2}-\left(\partial_{z} \phi\right)^{2}\right]
$$

where we have chosen a convenient normalization. In terms of $\widetilde{\phi}=e^{\frac{z m_{s}}{\sqrt{N}}} \phi$ it is

$$
S=\frac{(2 \pi)^{5}}{\kappa_{10}^{2}} \int d^{10} x\left[\left(\partial_{0} \widetilde{\phi}\right)^{2}-\left(D_{z} \widetilde{\phi}\right)^{2}\right]
$$

where $D_{z}=\partial_{z}-\frac{m_{s}}{\sqrt{N}}$. This action leads to the equation of motion

$$
\left(-\partial_{0}^{2}+\partial_{z}^{2}-\frac{m_{s}^{2}}{N}\right) \widetilde{\phi}=0
$$

corresponding to a free massive particle with mass $\frac{m_{s}}{\sqrt{N}}$. The two independent solutions in the tube are $\phi_{ \pm}=e^{\frac{m_{s}}{\sqrt{N}}\left(\beta_{ \pm}(s) z-i \sqrt{s} t\right)}$, with

$$
\beta_{ \pm}(s)=-1 \pm \sqrt{1-s}
$$

where

$$
s=\frac{\omega^{2} N}{m_{s}^{2}}
$$

is energy squared in units of the mass gap.

The string frame length of the tube is $\frac{\sqrt{N}}{m_{s}} \ln \left(\frac{\sqrt{N}}{g_{0}}\right)$, and goes to infinity as $g_{0} \rightarrow 0$. We set $g_{0}=0$ and then the asymptotic region is driven off to infinity, and can be ignored. Our geometry consists of an infinite tube capped at the bottom by $A d S_{7} \times S^{4}$.

A particle with $s<1$ cannot propagate in the tube and is confined in the AdS part of our geometry. A particle with $s>1$ can propagate in the tube, and can leak out of the AdS region into the tube. Consequently, absorption from the tube into the AdS region is also possible. 


\subsection{The Differential Equation}

When studying the near horizon region $r \ll \frac{\sqrt{N}}{m_{s}}$ it is convenient to rescale the coordinates, $\rho=\frac{r}{R_{11}}, \chi=\frac{x}{R_{11}} \cdot y_{i}=\frac{x_{i} m_{s}}{\sqrt{N}}$, where $i$ runs over the $5+1$ dimensions parallel to the brane. (2.2) becomes

$$
\begin{gathered}
d s^{2}=(N \widetilde{A})^{\frac{2}{3}} l_{p}^{2}\left[\widetilde{A}^{-1} d y_{6}^{2}+d \chi^{2}+d \rho^{2}+\rho^{2} d \Omega_{3}^{2}\right] \\
\widetilde{A}=\sum_{n=-\infty}^{\infty} \frac{\pi}{\left[\rho^{2}+(\chi-2 \pi n)^{2}\right]^{\frac{3}{2}}} .
\end{gathered}
$$

$\chi$ has periodicity $2 \pi$. The tube is the region $\rho \gg 1$, while $\rho \ll 1$ is the M5 part of the geometry.

The propagation of a complex minimally coupled scalar is governed by the action

$$
S_{b}=\frac{(2 \pi)^{5}}{\kappa_{11}^{2}} \int d^{11} x \sqrt{g}|\partial \phi|^{2} .
$$

We set $\phi=\theta e^{-i \omega t}=\theta e^{-i \sqrt{s} y_{0}}$ with $\theta$ constant on the 3 -sphere and the spatial directions parallel to the 5-brane. (2.11) becomes

$$
S_{b}=\frac{m_{s}^{6} V}{2 \pi} \int d \chi d \rho \rho^{3}\left(-\widetilde{A} s|\theta|^{2}+\left|\partial_{\chi} \theta\right|^{2}+\left|\partial_{\rho} \theta\right|^{2}\right)
$$

where $V=\int d^{6} x=\frac{N^{3}}{m_{s}^{6}} \int d^{6} y$ is the spacetime volume of the brane. $\theta$ obeys the equation of motion

$$
\partial_{\chi}^{2} \theta+\frac{1}{\rho^{3}} \partial_{\rho} \rho^{3} \partial_{\rho} \theta+\sum_{n=-\infty}^{\infty} \frac{s \pi}{\left[\rho^{2}+(\chi-2 \pi n)^{2}\right]^{\frac{3}{2}}} \theta=0
$$

which is valid for all $\rho$ and on the full complex $s$ plane. $s$ is real and positive in Minkowski space, and real and negative in Euclidean space. Recall that the mass gap in the tube is at $s=1$.

\subsection{A particular solution}

Define $f(s, \rho, \chi)$ as the unique solution to (2.13) obeying:

1. Near $\rho, \chi=0 f=f(s, u)$, where $u^{2}=\rho^{2}+\chi^{2}$. Further $f(s, u=0)=0$ in Euclidean space. This condition ensures that in Minkowski space at small $u, f$ is a wave that carries flux only into the 5 -branes. It uniquely determines $f(s, \rho, \chi)$ up to a normalization everywhere on the complex $s$ plane. In particular at small $u$

$$
f \approx C(s)\left(\frac{-s \pi}{u}\right)^{\frac{3}{2}} K_{3}\left(2 \sqrt{\frac{-s \pi}{u}}\right)
$$


2. $f$ is normalized such that for $\rho \gg 1$

$$
f(s, \rho, \chi) \approx \rho^{\beta_{+}(s)}+D(s) \rho^{\beta_{-}(s)} .
$$

$C(s), D(s)$ are determined in principle by (2.13). In the absence of a complete solution we list what we know about these two functions. Note that $C(s)$ and $D(s)$ are real on the negative real axis.

a. For real $s f(s, \rho, \chi)$ obeys a flux conservation equation derived from (2.13). The equation is trivial for negative $s$, but nontrivial for positive $s$ yielding

$$
\operatorname{Im}\left[(1+D(s))\left(1-D^{*}(s)\right) \sqrt{1-s}\right]=\frac{\pi^{3} s^{3}|C(s)|^{2}}{6} .
$$

b. In Appendix A we use the WKB approximation to argue that on the positive real axis

$$
\lim _{s \rightarrow \infty}|D(s)|=0 .
$$

c. At $s=0(2.13)$ becomes an equation for free propagation of waves in 4 spatial dimensions and may be exactly solved. $C(s)$ and $D(s)$ may then be determined for small $s$ by perturbing around this exact solution. In Appendix B we show that in perturbation theory $C(s)$ and $D(s)$ take the form (a similar result was anticipated in [5])

$$
\begin{aligned}
& C(s)=\sum_{n=0}^{\infty}\left(s^{3} \ln s\right)^{n} f_{n}(s) \\
& D(s)=\sum_{n=0}^{\infty}\left(s^{3} \ln s\right)^{n} g_{n}(s),
\end{aligned}
$$

where $f_{n}(s)$ and $g_{n}(s)$ are analytic functions at $s=0$ related to each other by (2.16). Explicitly computing the first few terms in the perturbative expansion (Appendix B) we find

$$
\begin{gathered}
f_{0}(s)=1+\frac{1}{2}[\gamma-\ln (4 \pi)] s+\mathcal{O}\left(s^{2}\right) \\
g_{0}(s)=\mathcal{O}\left(s^{2}\right) \\
f_{1}(s)=\frac{\zeta(3)}{48 \pi}+\mathcal{O}(s) \\
g_{1}(s)=\frac{\pi^{2}}{12}+\mathcal{O}(s)
\end{gathered}
$$

where $\gamma$ is Euler's constant. Therefore,

$$
\begin{gathered}
C(s)=1+\text { smaller terms, } \\
D(s)=\text { Analytic }+\frac{\pi^{2}}{12} s^{3} \ln s+\text { smaller terms } .
\end{gathered}
$$

To leading order $\operatorname{Im} D(s)=\frac{\pi^{3}}{12} s^{3}|C(s)|^{2}$ in accordance with (2.16). 


\section{Absorption from the tube for $s>1$}

When $s>1$ particles propagate in the tube. $f(s, \rho, \chi)$ for positive $s$ is a solution with unit flux incident down into the tube, flux $|D(s)|^{2}$ is reflected back up the tube, and flux $1-|D(s)|^{2}$ is absorbed into the M5-branes. Therefore, if a particle moves down the tube, the probability that it will be reflected back up the tube is $|D(s)|^{2}$. The reflection $S$ matrix element is $2 \sqrt{s-1} D(s)$ (see Appendix C for our normalization of the $S$ matrix).

Since particles with $s>1$ can be absorbed into the M5-branes, the reverse process the leakage of particles with $s>1$ out of the AdS region into the tube - is also possible. This is unlike the situation with a stack of M5-branes in flat space, which ceases to absorb and emit particles in the decoupling limit $l_{p} \rightarrow 0$.

This observation strengthens the Maldacena-Strominger [4] non-decoupling from the tube in two ways. First, the non-decoupling occurs at finite energy and does not require energy densities. Second, the non-decoupling occurs at energies above $\frac{m_{s}}{\sqrt{N}}$. This value is below $\frac{m_{s}}{N^{\frac{1}{6}}}$, which is required for the validity of the Maldacena-Strominger approximations.

\section{Two Point Functions}

\subsection{The Prescription}

In this section we will use the holographic proposal of [5] to find an expression for the two point function of the little string theory operator $O$ that couples to our minimal scalar according to

$$
S_{i n t}=\int d^{6} x\left(\theta^{*}(x, L) O(x)+\theta(x, L) O(x)^{*}\right) .
$$

Since $\theta$ is a mode of the graviton, we interpret $O$ as a component of the energy momentum tensor of the brane. We will be working in momentum space and use

$$
O(k)=\int \frac{d^{6} x}{(2 \pi)^{3}} O(x) e^{i k x}
$$

Let $\left\langle O(k) O^{*}\left(-k^{\prime}\right)\right\rangle=\Pi(k) \delta\left(k-k^{\prime}\right)$, and define a dimensionless two point function $\Pi^{L}(s)=$ $\frac{1}{m_{s}^{6}} \Pi(k)$ at $\frac{k^{2} N}{m_{s}}=s$.

$h(s, \rho, \chi)=\frac{f(s, \rho, \chi)}{f(s, L, \chi=0)}$ (for $L \gg 1$ the $\chi$ dependence of $f$ is exponentially small) is a solution of (2.13), which is regular everywhere in Euclidean space, and is unity at $\rho=L$. 
According to the prescription of [13,14] adapted to our situation, $\Pi^{L}$ may be found using the classical action (2.12) evaluated on the classical solution $h(s, \rho, \chi)$

$$
S_{b}[h(s, L, \chi)]=V m_{s}^{6} L^{3} \frac{\partial_{\rho} f(s, \rho, \chi)_{\rho=L}}{f(s, L, \chi)}
$$

We subtract from (4.2) the action evaluated on the 'free' solution $\left(\frac{\rho}{L}\right)^{\beta_{+}(s)}, S_{b}^{(0)}=$ $V m_{s}^{6} L^{3} \beta_{+}(s)$ and then retain only the dominant term as $L \rightarrow \infty$ (the toy model of Appendix C clearly motivates this prescription)

$$
\Pi^{L}(s)=L^{2}\left[\beta_{-}(s)-\beta_{+}(s)\right] D(s) L^{\beta_{-}(s)-\beta_{+}(s)}=L^{-2 \beta_{+}(s)} 2 \sqrt{1-s} D(s) .
$$

The renormalized correlation function is obtained by removing the $L$ dependence, and is given by

$$
\Pi(s)=2 \sqrt{1-s} D(s) .
$$

The renormalized correlation function thus defined agrees with the reflection $S$ matrix element computed in the previous section.

For small $s$ (4.4) becomes

$$
\Pi(s)=\text { analytic }+\frac{\pi^{2}}{6} s^{3} \ln s+\text { smaller terms. }
$$

This is exactly the two point function of the energy momentum tensor of the M5 theory, which couples to a massless minimally coupled scalar in $A d S_{7}$. This is a consistency check, since the IIA little string theory reduces to the $(0,2)$ theory at low energies.

\subsection{The implications of momentum dependent renormalizations}

In order to obtain a cut off $(L)$ independent $\Pi(s)$ from the bare two point function $\Pi^{L}(s)$ we had to perform a momentum dependent multiplicative renormalization. An additional finite momentum dependent renormalization would change the formula (4.4) for $\Pi(s)$. We have chosen our renormalization scheme to yield a two point function that agrees with the $S$ matrix computed in section 3. (Strictly, this might leave an $s$ dependent phase ambiguity.)

A momentum dependent renormalization is not multiplicative in position space. This may indicate the absence of a natural definition of the correlation function in position space, hinting at nonlocality of the theory [7,8] at scale $\frac{m_{s}}{\sqrt{N}}$. If correct, this effect must be distinct from the nonlocality at $m_{s}$ noted in [2]. 


\section{Absorption from Infinity}

In this section we consider NS5-branes in IIA theory with small but nonzero asymptotic string coupling $g_{0}$. The tube in the NS5-brane geometry is now of finite length, and the asymptotically flat part of the geometry cannot be ignored. We compute the absorption probability for a particle incident onto the NS5-brane from asymptotic infinity.

Consider a wave with $s>1$ incident (with zero momentum along the branes and the sphere) onto the 5-brane. This wave may be partially reflected at two locations - at the entrance to the tube from asymptotic infinity, with a reflection amplitude $R(s)$, and at the entrance to the AdS region from the tube, with a reflection amplitude $D(s)$.

In order to be absorbed a wave must penetrate the tube, and then either be absorbed, or be reflected an even number of times and then absorbed. Thus the absorption amplitude is

$$
A_{\infty}=\sqrt{\left(1-|D|^{2}\right)\left(1-|R|^{2}\right)} \sum_{n=0}^{\infty}\left(e^{i \gamma} D R\right)^{n} .
$$

$e^{i \gamma}=\left(\frac{\sqrt{N}}{g_{0}}\right)^{2 i \sqrt{s-1}}$ represents the phase picked up by the wave traversing twice the length of the tube. The absorption probability, $\mathcal{F}_{\infty}=\frac{\left(1-|D(s)|^{2}\right)\left(1-|R(s)|^{2}\right)}{\left|1-e^{i \gamma} D(s) R(s)\right|^{2}}$, is the ratio of flux absorbed by the 5 -branes and the flux incident on them from asymptotic infinity. $\mathcal{F}_{\infty}$ may be computed by matching an exact solution to the wave equation in the asymptotic and tube region with (2.15). We find

$$
\mathcal{F}_{\infty}=\frac{\left(1-|D(s)|^{2}\right)\left(1-e^{-2 \pi \sqrt{s-1}}\right)}{\left|1+\left(\frac{\sqrt{N}}{g_{0}}\right)^{2 i \sqrt{s-1}} D(s) e^{-\pi \sqrt{s-1}} \frac{\Gamma(\beta-(s)+2)}{\Gamma\left(\beta_{+}(s)+2\right)}\left(\frac{4}{s}\right)^{i \sqrt{s-1}}\right|^{2}},
$$

where $\Gamma$ is the Gamma function. In particular this implies $|R(s)|=e^{-\pi \sqrt{s-1}}$, in agreement with [15].

The absorption cross section of the 5-branes is related to the absorption probability by $\sigma_{\infty}=4 \pi \frac{\mathcal{F}_{\infty}}{\omega^{3}}$. Notice that, in contrast with a stack of M5-branes in flat space, the absorption cross section for a stack of NS5-branes is not zero in the limit $g_{0} \rightarrow 0\left(l_{p} \rightarrow 0\right)$. This may be understood as follows. A particle incident on a stack of isolated $M 5$ branes in flat space has to tunnel through a broad potential barrier extending (in the usual coordinates) from $r=\frac{1}{\omega}$ down to very small values of $r$. The suppression factor due to this barrier goes to infinity in the decoupling limit, so that, in order to send unit flux into the M5-branes one needs to shine an infinite amount of flux on them from infinity.

A stack of NS5-branes is, as we have seen, an array of stacks of M5-branes. The geometry very near any one element of the stack is identical to that of an M5 in flat space, 
but is significantly different for $r$ of order $R_{11}$. Since $R_{11} \rightarrow 0$ in the decoupling limit, most of the potential barrier present in the geometry of the isolated M5-brane is chopped off in this modified geometry, and is replaced by the tube, through which particles with $s>1$ propagate freely. The residual potential barrier results in only finite tunneling suppression even in the decoupling limit. In order to send unit flux down to the 5-branes, one needs to shine only a finite amount of flux onto the branes from infinity.

For completeness we present also the small $g_{0}$ flux absorption ratio $\mathcal{F}_{\infty}(s)$ for $s<1$

$$
\mathcal{F}_{\infty}=\left(\frac{g_{0}}{\sqrt{N}}\right)^{2 \sqrt{1-s}} \frac{\pi^{4}}{3} \frac{|C(s)|^{2}}{2^{2 \sqrt{1-s}}} \frac{s^{3+\sqrt{1-s}}}{|\Gamma(1+\sqrt{1-s})|^{2}} .
$$

The first factor in (5.2) is the tunneling suppression experienced by the $s<1$ particle in penetrating through the tube of length $\frac{g_{0}}{\sqrt{N}}$. Note that $\mathcal{F}_{\infty} \rightarrow 0$ as $g_{0} \rightarrow 0$.

\section{Acknowledgements}

We would like to acknowledge useful discussions with O. Aharony, M. Berkooz, T. Banks, C. Callan, O. Ganor, R. Gopakumar, I. Klebanov, S. Lee, J. Maldacena, S. Mathur, G. Moore, M. Rangamani, A. Vishwanath, E. Witten and especially A. Strominger. The work of S.M. was supported in part by DOE grant DE-FG02-91ER40671 and N.S. by DOE grant DE-FG02-90ER40542.

\section{Appendix A. Large $s$ Behavior}

In Minkowski space, for $u=\sqrt{\chi^{2}+\rho^{2}} \ll 1$ (2.13) describes the propagation of a wave whose coordinate wavelength $\delta u$ is approximately $\frac{u^{\frac{3}{2}}}{\sqrt{s}}$. The fractional change in length of a wavelength over the length of one wavelength, of order $\sqrt{\frac{u}{s}}$, is small for large $s$. Therefore, the WKB approximation is valid for $s \gg 1$, and the dynamics of (2.13) is that of null geodesics in $(2.10)$.

The geodesic equation for a particle moving in (2.10) with no velocity components along the $S^{3}$ and the brane is

$$
\begin{aligned}
& A y_{0}^{\prime \prime}-\frac{1}{3} \partial_{\rho} A \rho^{\prime} y_{0}^{\prime}-\frac{1}{3} \partial_{\chi} A \chi^{\prime} y_{0}^{\prime}=0 \\
& A \rho^{\prime \prime}+\frac{1}{3} \partial_{\rho} A\left(\rho^{\prime}\right)^{2}-\frac{1}{6 A} \partial_{\rho} A\left(y_{0}^{\prime}\right)^{2}-\frac{1}{3} \partial_{\rho} A\left(\chi^{\prime}\right)^{2}+\frac{2}{3} \partial_{\chi} A \rho^{\prime} \chi^{\prime}=0 \\
& A \chi^{\prime \prime}+\frac{1}{3} \partial_{\chi} A\left(\chi^{\prime}\right)^{2}-\frac{1}{6 A} \partial_{\chi} A\left(y_{0}^{\prime}\right)^{2}-\frac{1}{3} \partial_{\chi} A\left(\rho^{\prime}\right)^{2}+\frac{2}{3} \partial_{\chi} A \rho^{\prime} \chi^{\prime}=0
\end{aligned}
$$


where $y_{0}$ is the time coordinate in (2.10) and primes denote derivatives with respect to an affine parameter $\lambda$ along the curve. A null geodesic also satisfies the mass shell condition

$$
y_{0}^{\prime}=A \sqrt{\rho^{\prime 2}+\chi^{\prime 2}} .
$$

In the tube $A=\frac{1}{\rho^{2}}$ and (A.1), (A.2) admit the one parameter family of solutions

$$
\chi(\lambda)=\chi_{0} ; \quad \rho(\lambda)=\lambda^{\frac{3}{2}} ; \quad y_{0}(\lambda)=\frac{3}{2} \ln \lambda
$$

that describe a light ray traveling down that tube at a fixed value of $\chi=\chi_{0}$, where $-\pi<\chi_{0} \leq \pi$. Numerical integration of (A.1) into the AdS part of the geometry indicates that every geodesic in (A.3) except the one at $\chi_{0}=\pi$ reaches $\chi=0, \rho=0$ (and so is absorbed by the M5-brane) at a finite value of the affine parameter $\lambda_{0}$. As $\chi_{0} \rightarrow \pi, \lambda_{0} \rightarrow \infty$ and the geodesic at $\chi_{0}=\pi$ never reaches the horizon but is reflected back.

Hence, classically, a light ray propagating down the tube with no momentum along the $S^{3}$ or the brane is absorbed by the NS5-brane with unit probability and $D \approx 0$.

\section{Appendix B. Small $s$ Behavior}

We wish to determine $C(s)$ and $D(s)$ for small $s$. To do this we must find $f(s, \rho, \chi)$, the solution to (2.13) subject to the conditions listed in section 2.5.

At $s=0$ (2.13) has two linearly independent solutions that, for small $\rho, \chi$ are functions only of $u^{2}=\rho^{2}+\chi^{2}$. They are

$$
\psi_{1}=1, \quad \psi_{2}=\sum_{n=-\infty}^{\infty} \frac{\pi}{\left[\rho^{2}+(\chi-2 \pi n)^{2}\right]^{\frac{3}{2}}} .
$$

For large $\rho \psi_{2}=\frac{1}{\rho^{2}}$ and for small $u \psi_{2}=\pi\left(\frac{1}{u^{3}}+\frac{2 \zeta(3)}{(2 \pi)^{3}}\right)$.

$f(s=0, \rho, \chi)$ is a linear combination of $\psi_{1}$ and $\psi_{2}$. Regularity at $u=0$ sets the coefficient of $\psi_{2}$ to zero, and the normalization condition in sec 2.5 sets the coefficient of $\psi_{1}$ to unity. Therefore, $C(s=0)=1$ and $D(s=0)=0$.

We iterate our solution to successively higher order in $s$. Let

$$
\begin{gathered}
f=f_{0}+f_{1}+f_{2}+\ldots \\
C=C_{0}+C_{1}+C_{2}+\ldots
\end{gathered}
$$




$$
D=D_{0}+D_{1}+D_{2}+\ldots
$$

where $f_{n}, C_{n}, D_{n}$ are of successively of higher order in $s$ (later in this appendix we will find that $f_{n}, C_{n}, D_{n}$ are each of the form $s^{n} P_{n}(\ln s)$ where $P_{n}$ is a polynomial of degree $\left.\left[\frac{n}{3}\right]\right)$. Above we have found $f_{0}=\psi_{1}, C_{0}=1, D_{0}=0 . f_{n+1}$ is obtained from $f_{n}$ by solving (2.13) iteratively,

$$
-\left(\partial_{\chi}^{2}+\frac{1}{\rho^{3}} \partial_{\rho} \rho^{3} \partial_{\rho}\right) f_{n+1}=\left(\sum_{m=-\infty}^{\infty} \frac{s \pi}{\left[\rho^{2}+(\chi-2 \pi m)^{2}\right]^{\frac{3}{2}}}\right) f_{n} .
$$

(B.1) does not uniquely determine $f_{n+1}$, since the differential operator on the LHS of (B.1) has two zero modes, $\psi_{1}$ and $\psi_{2}$. At large $\rho f=\rho^{\beta_{+}(s)}+D(s) \rho^{\beta_{-}(s)}$ may be expanded as $f=1-s \frac{\ln \rho}{2}+\frac{s^{2}}{8}\left[(\ln \rho)^{2}-\ln \rho\right]+\ldots+\frac{1}{\rho^{2}}\left(D_{0}+D_{1}+D_{2}+\ldots\right)\left\{1+s \frac{\ln \rho}{2}+\frac{s^{2}}{8}\left[(\ln \rho)^{2}+\ln \rho\right]+\ldots\right\}$ and so the freedom to add an arbitrary multiple of $\psi_{1}$ to any solution of (B.1) is fixed by the condition that the coefficient of the constant term in an expansion of $f_{n+1}(\rho)$ at large $\rho$ is zero. Let $\theta_{n+1}$ be a particular solution of (B.1) obeying this asymptotic condition. Then

$$
f_{m+1}=\theta_{m+1}+B_{m+1} \psi_{2}
$$

where $B_{m+1}$ is a constant determined by imposing regularity of $f$ at small $u$. Regularity of $f$ at $u=0$ does not imply regularity of $f_{m}$ for any $m \neq 0$. It implies that $f_{m}$ is proportional to the appropriate term in the small $s$ expansion of $C(s)\left(\frac{-s \pi}{u}\right)^{\frac{3}{2}} K_{3}\left(2 \sqrt{\frac{-s \pi}{u}}\right)$. Expanding this order by order in $s$ we find

$$
f(s, \rho, \chi)=\left(C_{0}+C_{1}+C_{2} \ldots\right)\left\{1+\frac{s \pi}{2 u}+\frac{s^{2} \pi^{2}}{4 u^{2}}-\frac{s^{3} \pi^{3}}{12 u^{3}}\left[\ln \left(\frac{-s \pi}{u}\right)-\psi(1)-\psi(4)\right]+\ldots\right\} .
$$

Matching with this form fixes $B_{m+1}$ and $C_{m+1}$ at each order.

At first order we find

$$
f_{1}=s \lim _{M \rightarrow \infty}\left[\left(\sum_{n=-M}^{M} \frac{\pi}{2 \sqrt{\rho^{2}+(\chi-2 \pi n)^{2}}}\right)-\frac{1}{2} \ln (4 \pi M)\right] .
$$

At large $\rho f_{1}=-\frac{s}{2} \ln \rho$. At small $\rho f_{1}=s\left\{\frac{\pi}{2 u}+\frac{1}{2}[\gamma-\ln (4 \pi)]\right\}$ where $\gamma$ is Euler's constant. Therefore,

$$
C_{1}=\frac{s}{2}[\gamma-\ln (4 \pi)], \quad D_{1}=0
$$


This procedure may be iterated to higher orders.

$f_{i}, C_{i}, D_{i} \propto s^{i}$ for $i \leq 2$. Since $f_{2} \propto s^{2}$, it is possible to choose $\theta_{3} \propto s^{3}$. However, at small $u f_{3} \approx s^{3} h(u)+\frac{\pi^{3}}{12 u^{3}} s^{3} \ln s$, and therefore, we must choose $B_{3}=A s^{3}+\frac{\pi^{2}}{12} s^{3} \ln s$, where $A$ is a constant. This implies $D_{3}=($ const $) s^{3}+\frac{\pi^{2}}{12} s^{3} \ln s$ and $C_{3}=($ const $) s^{3}+\frac{\zeta(3)}{48} s^{3} \ln s$.

Since at small $u f_{3}=s^{3} h(u)+\frac{\pi^{3} s^{3} \ln s}{12 u^{3}}$, it is possible to choose $\theta_{4}=g(u) s^{4}+s^{4} \ln s\left(\frac{c_{1}}{u}+\right.$ $\left.\frac{c_{2}}{u^{4}}+c_{3}\right)$ for small $u$. To ensure matching with (B.2) each of $C_{4}, B_{4}$ must contain a term proportional to $s^{4} \ln s$. The situation is similar at the next order. However, at sixth order, matching with (B.2) (specifically the cross term from $C_{3}$ and the $\frac{1}{u^{3}}$ term) forces us to include also a term proportional to $s^{6}(\ln s)^{2}$ in $B_{6}$, and hence in $C_{6}, D_{6}$.

Continuing successively to higher orders, it is clear that $D_{i}$ and $C_{i}$ are of the form $s^{i} P_{i}(\ln s)$, where $P_{i}(x)$ is a polynomial of order $\left[\frac{i}{3}\right]$ in $x$. In other words, at small $s, D(s)$ and $C(s)$ may each be written as a power series in $s$ and $s^{3} \ln s$.

\section{Appendix C. A Toy Model}

In the string frame, the near horizon geometry of the NS5-brane is a semi-infinite tube, connected through an intermediate region to the M5-brane geometry. A quantum incident down the tube is either reflected back up the tube, or continues through the horizon of the 5-brane and disappears.

In some ways this problem is similar to a free scalar field theory with the action

$$
S=\frac{1}{2} \int d x d t\left\{\left(\partial_{0} \phi\right)^{2}-\left(\partial_{x} \phi\right)^{2}-\theta(-x) \phi^{2}\right\}
$$

in $1+1$ dimensions. The region $x<0$ is analogous to the tube, $x>0$ is similar to the M5 region, while the abrupt change in mass at $x=0$ is the analogue of the transition region between the NS5 tube and the M5 region.

In this Appendix we explore this toy model. We compute the $S$ matrix of this model in three different ways: using the LSZ formula, computing flux ratios, and computing the Euclidean action as a function of boundary values. We then consider a holographic projection of this theory, and demonstrate that the two point function of the boundary operator is equal to the reflection $S$ matrix of the bulk particle. 


\section{C.1. The Propagator}

The Euclidean space propagator

$$
G(x, y, s)=-\int d t e^{i\left(t-t^{\prime}\right) \sqrt{-s}}\left\langle\phi(x, t) \phi\left(y, t^{\prime}\right)\right\rangle
$$

is easily found by solving the appropriate differential equation with the boundary condition that it vanishes as either $|x|$ or $|y|$ go to infinity. Our conventions are such that $s$ is real and positive for Lorentzian energies and real and negative for Euclidean energies. We find

$$
G(x, y, s)= \begin{cases}G^{0}(x-y, s, m=1)+e^{\sqrt{1-s}(x+y)} A(s) & x, y \leq 0 \\ e^{\sqrt{1-s} x-\sqrt{-s} y} B(s) & x \leq 0 ; y \geq 0 \\ G^{0}(x, y, s, m=0)+e^{-\sqrt{-s}(x+y)} \widetilde{A}(s) & x, y \geq 0\end{cases}
$$

where

$$
\begin{aligned}
& A(s)=-\frac{1}{2 \sqrt{1-s}}(1-2 s-2 \sqrt{-s} \sqrt{1-s}) \\
& B(s)=\sqrt{-s}-\sqrt{1-s} \\
& \widetilde{A}(s)=\frac{1}{2 \sqrt{-s}}(1-2 s-2 \sqrt{-s} \sqrt{1-s})
\end{aligned}
$$

and

$$
G^{0}(x-y, s, m)=\frac{-1}{2 \pi} \int_{-\infty}^{\infty} d p \frac{e^{i p(x-y)}}{-s+p^{2}+m^{2}}=\frac{-e^{-\sqrt{m^{2}-s}|x-y|}}{2 \sqrt{m^{2}-s}}
$$

is a free propagator. $G$ can also be written as

$$
\begin{aligned}
& G(x, y, s)= \\
& \begin{cases}G^{0}(x-y, s, m=1)+G^{0}(x-0, s, m=1) G^{0}(0-y, s, m=1) \Gamma_{L L}(s) & x, y \leq 0 \\
G^{0}(x-0, s, m=1) G^{0}(0-y, s, m=0) \Gamma_{L R}(s) & x \leq 0 ; y \geq 0 \\
G^{0}(x, y, s, m=0)+G^{0}(x-0, s, m=0) G^{0}(0-y, s, m=0) \Gamma_{R R}(s) & x, y \geq 0\end{cases}
\end{aligned}
$$

in terms of

$$
\begin{aligned}
& \Gamma_{L L}=-2((1-2 s) \sqrt{1-s}-2(1-s) \sqrt{-s}) \\
& \Gamma_{L R}=-4((1-s) \sqrt{-s}+s \sqrt{1-s}) \\
& \Gamma_{R R}=2((1-2 s) \sqrt{-s}+2 s \sqrt{1-s}) .
\end{aligned}
$$

Equations (C.3)-(C.7) are valid by analytic continuation on the complex $s$ plane with square root functions defined as follows. For $s=X e^{i \alpha}(0 \leq \alpha<2 \pi)$ we set $\sqrt{-s}=$ $-i \sqrt{s}=\sqrt{X} e^{i \frac{\alpha-\pi}{2}}$. Similarly for $s-1=X e^{i \alpha}$ we set $\sqrt{1-s}=-i \sqrt{s-1}=\sqrt{X} e^{i \frac{\alpha-\pi}{2}}$. In 
particular, for $s$ infinitesimally above the real axis (C.3) is the Minkowskian propagator $G_{M}$ defined by

$$
i G_{M}(x, y, s)=\int e^{i \sqrt{s} x^{0}}\left\langle T \phi\left(x, x^{0}\right) \phi(y, 0)\right\rangle_{\text {Minkowski }} d x^{0} .
$$

\section{C.2. $S$ Matrix from the Propagator}

When $s>1$ we have two kinds of in states: particles with momentum $p_{i n}=\sqrt{s-1}>$ 0 coming from the left and particles with momentum $k_{i n}=-\sqrt{s}<0$ coming from the right. We also have two kinds of out states: particles with momentum $p_{\text {out }}=-\sqrt{s-1}<0$ going to the left and particles with momentum $k_{\text {out }}=\sqrt{s}>0$ going to the right. We normalize states covariantly so that $\left\langle k_{i n} \mid k_{i n}^{\prime}\right\rangle=2 \sqrt{s} \delta\left(\sqrt{s_{i n}}-\sqrt{s_{i n}^{\prime}}\right),\left\langle p_{i n} \mid p_{i n}^{\prime}\right\rangle=2 \sqrt{s-1} \delta\left(\sqrt{s_{i n}}-\right.$ $\left.\sqrt{s_{i n}^{\prime}}\right)$, and similarly for out states. The covariant $S$ matrix with this normalization of states is obtained by amputating the external propagators from (C.6). It is given by

$$
\left(\begin{array}{cc}
\left\langle p_{\text {out }} \mid p_{\text {in }}\right\rangle & \left\langle p_{\text {out }} \mid k_{\text {in }}\right\rangle \\
\left\langle k_{\text {out }} \mid p_{\text {in }}\right\rangle & \left\langle k_{\text {out }} \mid k_{\text {in }}\right\rangle
\end{array}\right)=\delta\left(\sqrt{s_{\text {in }}}-\sqrt{s_{\text {out }}}\right)\left(\begin{array}{cc}
\Gamma_{L L}(s) & \Gamma_{L R}(s) \\
\Gamma_{L R}(s) & \Gamma_{R R}(s)
\end{array}\right) .
$$

With our normalization, completeness of in states implies

$$
\int_{0}^{1} d \sqrt{s} \frac{\left|k_{i n}\right\rangle\left\langle k_{i n}\right|}{2 \sqrt{s}}+\int_{1}^{\infty} d \sqrt{s}\left(\frac{\left|p_{i n}\right\rangle\left\langle p_{i n}\right|}{2 \sqrt{s-1}}+\frac{\left|k_{i n}\right\rangle\left\langle k_{i n}\right|}{2 \sqrt{s}}\right)=1 .
$$

Inserting (C.9) into the the scalar product between two arbitrary in states we verify that the $S$ matrix is unitary

$$
\left(\begin{array}{cc}
\Gamma_{L L}^{*}(s) & \Gamma_{L R}^{*}(s) \\
\Gamma_{L R}^{*}(s) & \Gamma_{R R}^{*}(s)
\end{array}\right)\left(\begin{array}{cc}
\frac{1}{2 \sqrt{s-1}} & 0 \\
0 & \frac{1}{2 \sqrt{s}}
\end{array}\right)\left(\begin{array}{ll}
\Gamma_{L L}(s) & \Gamma_{L R}(s) \\
\Gamma_{L R}(s) & \Gamma_{R R}(s)
\end{array}\right)\left(\begin{array}{cc}
\frac{1}{2 \sqrt{s-1}} & 0 \\
0 & \frac{1}{2 \sqrt{s}}
\end{array}\right)=1 .
$$

For $s<1$ all particles incident from the right are reflected, and the appropriately normalized $S$ matrix is a pure phase.

\section{C.3. $S$ matrix through flux ratios}

The probability for a particle incident from the left at energy $\sqrt{s}$ to continue thorough to $x=\infty, \mathcal{F}$, may be computed very simply. A purely in-going wave function for $x>0$ is

with

$$
\psi(x)= \begin{cases}e^{i p x}+E(s) e^{-i p x} & x \leq 0 \\ F(s) e^{i k x} & x \geq 0\end{cases}
$$

$$
F=\frac{2 \sqrt{s-1}}{\sqrt{s}+\sqrt{s-1}}=1+E, \quad E=\frac{-\sqrt{s}+\sqrt{s-1}}{\sqrt{s}+\sqrt{s-1}}
$$

and hence

$$
\mathcal{F}=1-|E|^{2}=\frac{4 \sqrt{s} \sqrt{s-1}}{(\sqrt{s}+\sqrt{s-1})^{2}} .
$$

This result is equivalent to the $S$ matrix of the previous section. For instance, given (C.8), the amplitude for reflection is $\frac{\Gamma_{L L}}{2 \sqrt{s-1}}=E(s)$ (where we have accounted for state normalizations and the delta function). 


\section{C.4. $S$ matrix from the Euclidean Action}

The scattering solution used in the flux computation of the previous section may be analytically continued to the complex $s$ plane

$$
\psi(x)= \begin{cases}e^{-\sqrt{1-s} x}+E(s) e^{\sqrt{1-s} x} & x \leq 0 \\ F(s) e^{-\sqrt{-s} x} & x \geq 0\end{cases}
$$

where

$$
F(s)=\frac{2 \sqrt{1-s}}{\sqrt{-s}+\sqrt{1-s}} ; \quad E(s)=\frac{-\sqrt{-s}+\sqrt{1-s}}{\sqrt{-s}+\sqrt{1-s}} .
$$

For $s$ real and negative we compute the action for this solution on $-L<x<\infty$.

$$
S=\frac{\partial \psi}{\psi}_{x=-L}=\sqrt{1-s} \frac{e^{-2 \sqrt{1-s} L} E(s)-1}{e^{-2 \sqrt{1-s} L} E(s)+1} .
$$

In terms of the action $S_{0}$ of the similar solution of a free massive theory $\psi=e^{-\sqrt{1-s} x}$

$$
\lim _{L \rightarrow \infty} \frac{\left(S-S_{0}\right)}{e^{-2 \sqrt{1-s} L}}=\Gamma_{L L}(s) .
$$

Thus after a subtraction and renormalization, the Euclidean action reproduces the reflection $S$ matrix.

\section{C.5. Effective theory on the boundary}

Consider a distinct but related physical theory in which a free particle of unit mass on the real line interacts with an operator $O$ in a quantum mechanical system situated at $x=-L$ via the interaction action

$$
S_{i n t}=\int d t \phi(-L, t) O(t) .
$$

We will look for an $O$ such that the $\phi(x, t)$ Greens functions computed at $x, y<-L$ are identical to (C.6).

The Euclidean space $\phi$ Greens function is given by

$$
\begin{aligned}
& \widetilde{G}(x, y, t)=-\left.\frac{\delta}{\delta J(x, t)} \frac{\delta}{\delta J(y, 0)} \int \mathcal{D} \phi e^{-S_{f}(\phi)+\int d \tau \phi(-L, \tau) O(\tau)+\int d \tau d \chi J(\chi, \tau) \phi(\chi, \tau)}\right|_{J=0} \\
& =G^{0}(x-y, t, m=1)-\int d t^{\prime} d \tau G^{0}(x+L, t-\tau, m=1) G^{0}\left(-L-y, t^{\prime}, m=1\right)\left\langle O(\tau) O\left(t^{\prime}\right)\right\rangle
\end{aligned}
$$


$\left(S_{f}(\phi)\right.$ is the free Euclidean action for the $\phi$ field) provided all higher $n$ point functions of $O$ vanish. Setting $\widetilde{G}(x, y, s)=\int d t e^{i \sqrt{s} t} \widetilde{G}(x, y, t)$ we find

$$
\begin{aligned}
\widetilde{G}(x, y, s) & =G^{0}(x-y, s, m=1) \\
& -G^{0}(x-0, s, m=1) G^{0}(0-y, s, m=1) e^{2 \sqrt{1-s} L} \int d t e^{\sqrt{-s}}\langle O(t) O(0)\rangle .
\end{aligned}
$$

We choose

$$
\int d t e^{\sqrt{-s} t}\langle O(t) O(0)\rangle=-e^{-2 \sqrt{1-s} L} \Gamma_{L L}(s)=S_{0}-S .
$$

This ensures $\widetilde{G}(x, y, s)=G(x, y, s)$, and the dynamics of $\phi(x, t)$ for $x<-L$ in the new system is identical to the dynamics of $\phi$ governed by (C.1) for $x<-L$. It should be stressed that there is no simple quantum mechanical system with such correlation functions of $O$.

\section{Appendix D. Geodesic Completion of the Brane Metrid}

In this appendix we describe the geodesic completion of the near horizon metric of the NS5-brane. As a preliminary, following [16], we describe the completion of the full (not merely near horizon) geometry of M2, M5 and D3 branes, and demonstrate that the completion of the geometry of several separated M5-branes is regular.

\section{D.1. Coincident extremal M2,M5 and D3 branes 10}

The metric of a single wedge in the geometry of a set of coincident M2, M5 or D3 branes is

$$
d s^{2}=A^{-\frac{2}{p+1}} d x_{p+1}^{2}+A^{\frac{2}{d-2}}\left(d r^{2}+r^{2} d \Omega_{d-1}^{2}\right)
$$

$(0<r<\infty)$, where $p$ is the spatial dimension of the brane, $d$ the spatial dimension of the transverse space, and $A=1+\left(\frac{\Lambda}{r}\right)^{d-2}$. The Penrose diagram for this patch of the geometry is a diamond whose edges on the right are $\mathcal{I}^{ \pm}$and those to the left are horizons at $t= \pm \infty$, at finite affine distance.

In terms of $\zeta=K r^{\frac{p+1}{d-2}}$ (where $K$ is a constant) the metric of the near horizon region $\zeta^{p+1} \ll \Lambda^{d-2}$ of (D.1) is

$$
d s^{2}=\Lambda^{2}\left(\frac{p+1}{d-2}\right)^{2}\left(\zeta^{2} d x_{p+1}^{2}+\frac{d \zeta^{2}}{\zeta^{2}}\right)+\Lambda^{2} d \Omega_{d-1}^{2} .
$$

5 This appendix was worked out in collaboration with A. Strominger. 
(D.2) may be smoothly continued past its horizons by extending the range of $\zeta$ to negative values, hence the same is true of (D.1) (written in terms of $\zeta$ ) for $|\zeta| \ll K \Lambda^{\frac{p+1}{d-2}}$. For larger $|\zeta|$ the Harmonic function $A=1+\frac{\Lambda^{d-2}}{\zeta^{p+1}}$ behaves differently for odd and even $p . A$ is well behaved for all $\zeta$ when $p$ is odd, but vanishes at $\zeta=-\Lambda^{\frac{d-2}{p+1}}$ when $p$ is even, leading to a curvature singularity in (D.1) at that point.

The Penrose diagram of (D.1) is a diamond. Depending on whether $p$ is even or odd our extension of the geometry augments the diamond differently. For $p$ odd we add another diamond whose bottom right edge is attached to the top left edge of the original diamond. The two left edges of the new diamond are new $\mathcal{I}^{ \pm}$. The upper right edge is a new horizon at finite affine distance. For $p$ even we add a triangle, whose bottom right edge is attached to the top left edge of the original diamond. The top right edge is a new horizon at finite affine distance. The vertical line is the singularity. In both cases the extension to the original geometry has its own horizon at finite affine distance, which may in turn be continued through. The corresponding Penrose diagrams are depicted in fig. 2. Each point on the diagram represents $S^{d-1} \times R^{p}$.

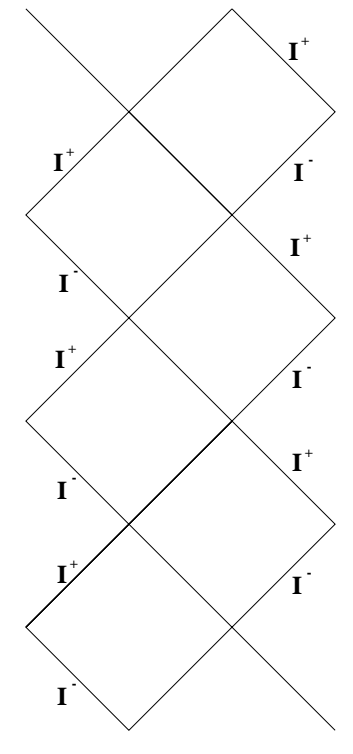

$\mathbf{a}$

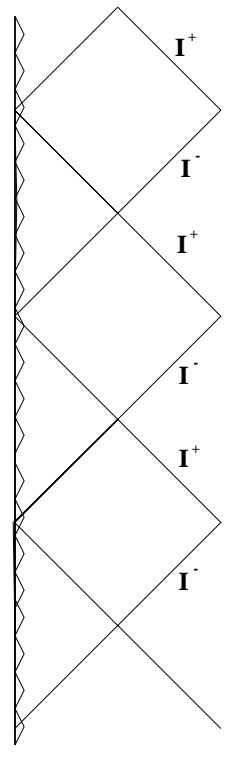

b

Fig. 2: Penrose diagram of the geodesic completion of the metric of the M5 and D3 brane (2a), and the M2 brane (2b). (2a) is also the Penrose diagram for a $\chi=0$ slice of the near horizon region of the NS5-brane.

\section{D.2. Multiple M5 geometry 10]}

The metric corresponding to two non-coincident $p$ branes is of the form (D.1). The harmonic function $A$ however picks up an additional term corresponding to the second 
brane. Let the first brane be located at the origin, and the second brane on the $z$ axis at $z=a$ in a Cartesian coordinate system in transverse space. The term in $A$ corresponding to the second brane is (recall $r=K \zeta^{\frac{p+1}{d-2}}$ )

$$
A=\frac{\Lambda^{d-2}}{\left(K^{2} \zeta^{\frac{2(p+1)}{d-2}}-2 K a \cos \theta \zeta^{\frac{p+1}{d-2}}+a^{2}\right)^{\frac{d-2}{2}}}, \quad \sin \theta=\frac{z}{r} .
$$

We attempt to extend through the horizon of the first brane by extending the range of $\zeta$ to negative values. This is permissible only if $\frac{p+1}{d-2}$ is an integer (in order for (D.3) to be real). If $\frac{p+1}{d-2}$ is moreover an even integer, as is the case for M5-branes, then the "mirror universe' $(\zeta<0)$ is identical to the original in every respect. Therefore, this is also true of any further extension through any other brane. The full space is completely regular.

\section{D.3. The IIA NS5-brane}

Consider an array of M5-branes periodically identified. The identification is regular in each wedge as every wedges has the geometry of (2.2). Since wedges in the multiple M5 geometry may be patched together in a regular manner we conclude that the full geometry is regular.

We focus on the near horizon region of the NS5-brane geometry. The geodesic completion of a $\chi=0$ slice of (2.10) has the Penrose diagram depicted in fig. $2 \mathrm{a}$, where $\mathcal{I}^{ \pm}$ represent light-like asymptotic infinity in the NS5-brane tube. As demonstrated in appendix A, null geodesics starting out in the tube at arbitrary values of $\chi$ are qualitatively similar to those starting at $\chi=0$, and so fig. 2a provides a fair picture of the causal structure of the spacetime. Each point in the diagram may roughly be thought of as the product of $R^{5}$, an $S^{3}$ and an $S^{1}$. The $S^{1}$ shrinks to zero size at the boundaries of the diagram. 


\section{References}

[1] C. Callan, J. Harvey and A. Strominger, "Supersymmetric String Solitons," hepth/9112030, Lectures at the 1991 Trieste Spring School on String Theory and Quantum Gravity.

[2] N. Seiberg, "New Theories in Six Dimensions and Matrix Description of M-theory on $T^{5}$ and $T^{5} / Z_{2}, "$ Phys. Lett. B408 (1997) 98, hep-th/9705221.

[3] M. Berkooz, M. Rozali and N. Seiberg, "Matrix description of M theory on $T^{4}$ and $T^{5}$," Phys. Lett. B408 (1997) 105, hep-th/9704089.

[4] J. Maldacena and A. Strominger, "Semiclassical decay of near extremal fivebranes," JHEP 12 (1997) 008, hep-th/9710014.

[5] O. Aharony, M. Berkooz, D. Kutasov and N. Seiberg, "Linear Dilatons, NS5-branes and Holography," JHEP 10 (1998) 004, hep-th/9808149.

[6] J. Maldacena, "The large N limit of Superconformal theories and Supergravity," Adv.Theor.Math.Phys. 2 (1998) 231, hep-th/9711200.

[7] A. Peet and J. Polchinski, "UV/IR Relations in AdS Dynamics," Phys.Rev. D59 (1999) 65006, hep-th/9809022.

[8] O. Aharony and T. Banks, "Note on the Quantum Mechanics of M Theory," JHEP 03 (1999) 016, hep-th/9812237.

[9] O. Aharony, M. Berkooz, S. Kachru, N. Seiberg, E. Silverstein, "Matrix Description of Interacting Theories in Six Dimensions," Adv.Theor.Math.Phys. 1 (1998) 148, hepth/9707079.

[10] E. Witten, "On The Conformal Field Theory Of The Higgs Branch,", JHEP 07 (1997) 003, hep-th/9707093.

[11] N. Itzhaki, J.M. Maldacena, J. Sonnenschein and S. Yankielowicz, "Supergravity and the Large $N$ Limit of Theories with Sixteen Supercharges," Phys.Rev. D48 (1998) 46, hep-th/9802042.

[12] S. Gubser, I. Klebanov, A. Tseytlin, "String Theory and Classical Absorption by Threebranes," Nucl.Phys. B499 (1997) 217, hep-th/9703040.

[13] S. Gubser, I. Klebanov and A. Polyakov, "Gauge theory Correlators from Non-Critical String Theory," Phys.Lett. B428 (1998) 105, hep-th/9802109.

[14] E. Witten, "Anti De Sitter Space And Holography," Adv.Theor.Math.Phys. 2 (1998) 253, hep-th/9702150.

[15] S. Gubser and I. Klebanov, "Absorption by branes and Schwinger terms in the World Volume Theory," Phys.Lett. B413 (1997) 41, hep-th/9708005.

[16] G. Gibbons, G. Horowitz and P. Townsend, "Higher-dimensional resolution of dilatonic black hole singularities," Class.Quant.Grav. 12 (1995) 297, hep-th/9410073. 